\title{
PRESENTACIÓN
}

\section{Genómica y proteómica en enfermedades infecciosas}

T as enfermedades infecciosas ocasionan más de una cuarta parte de las defunciones a nivel mundial. En particular, seis enfermedades (VIH/SIDA, tuberculosis, diarreas, paludismo, sarampión y neumonías) ocasionan 90\% de estas muertes. La magnitud real del problema está subestimada, particularmente en países en desarro1lo, donde muchas muertes por causas infecciosas no se clasifican como tales, tal como ocurre con las muertes por neoplasias secundarias a agentes infecciosos, como los virus de papiloma humano y hepatitis B y C.

Es de importancia para la salud pública el estudio de las infecciones nuevas, reemergentes o resistentes a antimicrobianos, cuya frecuencia ha aumentado de manera alarmante en las últimas dos décadas. A partir de la década de los noventa han aparecido agentes nuevos como el coronavirus causante del SARS; se han extendido enfermedades como la fiebre por virus del Nilo Occidental, la influenza, el cólera o el dengue o se han usado agentes tales como el ántrax para causar ataques bioterroristas. Por otra parte, con el desciframiento del genoma humano y el genoma de diversos agentes patógenos, el avance de la bioinformática y el mejoramiento de diversas técnicas moleculares, como la secuenciación del ADN y la proteómica, ahora se dispone de herramientas muy potentes para obtener información útil y enfrentar brotes o epidemias de enfermedades infecciosas con mayor éxito.

Todo lo anterior subraya la importancia de estar preparados para el estudio de las enfermedades infecciosas en el contexto de la salud pública. Muestra de ello es el caso de México, que puso a prueba su capacidad asistencial e investigativa en salud pública al ser el primer país en presentar un comportamiento epidémico de influenza A H1N1 de origen porcino. Dada la situación actual, es necesario aplicar los conocimientos adquiridos sobre la magnitud, determinantes y consecuencias de la propagación de los agentes infecciosos en la evaluación epidemiológica y biomédica, así como en la planeación estratégica y la toma de decisiones con evidencia científica orientadas al desarrollo de políticas y la regulación de las enfermedades infecciosas.
El Instituto Nacional de Salud Pública, y en particular el Centro de Investigación sobre Enfermedades Infecciosas (CISEI), cuenta con una reconocida trayectoria en el campo de la investigación de este tipo de enfermedades. Su capacidad académica y el avance logrado en las nuevas tecnologías han sido las bases para la preparación de este suplemento especial: Genómica y proteómica en enfermedades infecciosas en la revista Salud Pública de México.

En este suplemento hemos seleccionado doce artículos de revisión actualizada sobre genómica y proteómica de las enfermedades infecciosas. Los cinco primeros se concentran en aspectos generales de la genómica y proteómica en salud pública, así como su aplicación en estudios de epidemiología genética, nutrigenética, farmacogenómica y diversas aplicaciones de la proteómica. Estas estrategias moleculares han sido usadas para el diagnóstico de enfermedades infecciosas, determinar la participación genética en enfermedades complejas con un componente infeccioso y el desarrollo más acelerado de las vacunas.

Los dos primeros artículos son de carácter introductorio a la genómica y a la proteómica, respectivamente. En "La Medicina genómica aplicada a la salud pública", Ana Burguete, Víctor Bermúdez Morales y Vicente Madrid Marina describen la visión de la medicina genómica como una disciplina científica encargada del mapeo, secuenciación y análisis de los genomas. La identificación y entendimiento de los genes de los organismos han generado un amplio conocimiento de la estructura y la función de los genomas y han servido para desarrollar estrategias que pueden ser aplicadas en el control y prevención de las enfermedades infecciosas. En el segundo artículo, "La proteómica como herramienta en la investigación en salud pública", Victoria Pando y Humberto Lanz Mendoza abordan el tema desde la perspectiva del desarrollo de una medicina personalizada, que es complementaria al estudio de polimorfismos en el ADN. En conjunto esto permitirá la prevención y control de muchas enfermedades. 
Los tres artículos siguientes forman parte de un grupo de revisión de aspectos generales para comprender fundamentos sobre patogenia y diagnóstico de los agentes infecciosos, así como para el desarrollo de vacunas. Fidel de la Cruz Hernández Hernández y Mario Henry Rodríguez, en el artículo "Avances Biotecnológicos en el diagnóstico de enfermedades infecciosas", revisan los nuevos desarrollos en biotecnología y la ingeniería molecular de reactivos que han facilitado la producción a gran escala de reactivos de alta pureza, el desarrollo de pruebas más sensibles con alta especificidad y la automatización para el procesamiento simultáneo de un número grande de muestras. Por otro lado, Jesús Hernández Romano, Jesús Martínez Barnetche y Verónica Valverde Garduño escribieron "Polimorfismos reguladores y su papel en la patogenia de enfermedades complejas en la era postgenómica", donde resaltan que el estudio de los polimorfismos de un solo nucleótido, que afectan la expresión genética, está cobrando mayor interés, en virtud de que las diferencias de la expresión genética entre individuos pueden influir fenotípicamente en la susceptibilidad o la resistencia a enfermedades infecciosas, así como la severidad de sus manifestaciones clínicas. El último artículo de este grupo, de Javier Mota Sánchez, "Vacunas de ADN: inducción de la respuesta inmune", describe cómo con tecnología de punta en el área de la genómica y la proteómica es posible acelerar el desarrollo de nuevas vacunas con características mejoradas en aspectos fundamentales como la inmunogenicidad y la seguridad.

En el siguiente grupo de artículos, cuatro de ellos analizan en forma particular la aplicación de estas nuevas ciencias en el avance del conocimiento de microorganismos específicos, así como los eventos moleculares que determinan la resistencia bacteriana ante los antimicrobianos. Así, Ivone Castro y María del Carmen Rodríguez, en el artículo "Análisis proteómico de Plasmodium, el agente causal de la malaria", hacen un análisis de los hallazgos más recientes de la investigación proteómica del parásito causante del paludismo, para conocer con exactitud el papel que desempeñan estas proteínas en la transmisión. Jorge Falcón Lezama, Gilma Sánchez Burgos y José Ramos Castañeda, en la "Genética de poblaciones virales y transmisión de Dengue", resaltan lo mucho que se ha avanzado, con el desarrollo de la genómica y la bioinformática, para la identificación de determinantes moleculares de la transmisión, lo que ha permitido establecer estrategias de control y vigilancia eficaces. Janine Ramsey y colaboradores, en las "Contribuciones de la genética al estudio de la enfermedad de Chagas", enfatizan que el conocimiento sobre el genoma y el proteoma del Trypanosoma será esencial para desarrollar herramientas precisas y eficaces a corto y largo plazo para la prevención de la transmisión. Ulises Garza Ramos, Jesús Silva Sánchez, y Esperanza Martínez Romero, en el trabajo "Genética y genómica de la resistencia bacteriana, en particular de las $\beta$-lactamasas", describen el uso de la genómica para la identificación de nuevos blancos moleculares para inhibir el crecimiento de bacterias patógenas, lo que permitirá el diseño racional de nuevas drogas.

Finalmente, los últimos tres artículos versan sobre temas específicos de la aplicación de estas nuevas tecnologías para la identificación de inmunógenos que son usados para el desarrollo de vacunas, para el estudio de la epidemiología genética de la tuberculosis y para el desarrollo de nuevos sistemas de diagnóstico molecular del papilomavirus humano en esta nueva era de las vacunas profilácticas contra este virus. Germán Aguilar Gutiérrez y colaboradores escribieron la "Relevancia de la virulencia de factores de Helicobacter pylori para el desarrollo de vacunas", donde enfatizan que el desarrollo de una vacuna que confiera protección inmune a largo plazo es la mejor estrategia para su erradicación, y que la genómica y proteómica son verdaderas herramientas para este propósito. El grupo de investigación del Consorcio Mexicano contra la Tuberculosis, encabezado por Lourdes García García, escribió el trabajo "Epidemiología convencional y molecular en Orizaba, Veracruz, 1996-2006", donde se describen aspectos generales y particulares de la epidemia de tuberculosis en esa región de México y detallan cómo con ayuda de las ciencias genómica y proteómica pudieron medir la carga de la enfermedad, las consecuencias de la farmacorresistencia y desarrollar técnicas diagnósticas. El último artículo, “Diagnóstico molecular del papilomavirus humano en el desarrollo del cáncer cervical", escrito por Lourdes Gutiérrez Xicoténcatl, Tanya Plett Torres, Claudia Madrid González y Vicente Madrid Marina, describe las aproximaciones moleculares más fructíferas para este propósito.

Estas tecnologías exitosas han revolucionado la investigación científica y han permitido el uso de las mismas para contribuir en el control de brotes y epidemias por agentes infecciosos en los países en desarrollo. Como ejemplos de estas aplicaciones tecnológicas están la reacción en cadena de la polimerasa en tiempo real para la detección del virus de influenza AH1N1 y la implementación de pruebas moleculares de tamizaje de alta fidelidad para la detección del ADN de papilomavirus humano en la población más marginada. Esto ilustra el potencial que poseen estas tecnologías para la respuesta oportuna del sector salud en beneficio de la salud pública de nuestro país y de la región.

Dr. Vicente Madrid-Marina, ${ }^{(1)}$
Dr. Humberto Lanz-Mendoza, ${ }^{(1)}$
Dr. Mario Henry Rodríguez, ${ }^{(1)}$

